\title{
Landmark-free, parametric hypothesis tests regarding two- dimensional contour shapes using coherent point drift registration and statistical parametric mapping
}

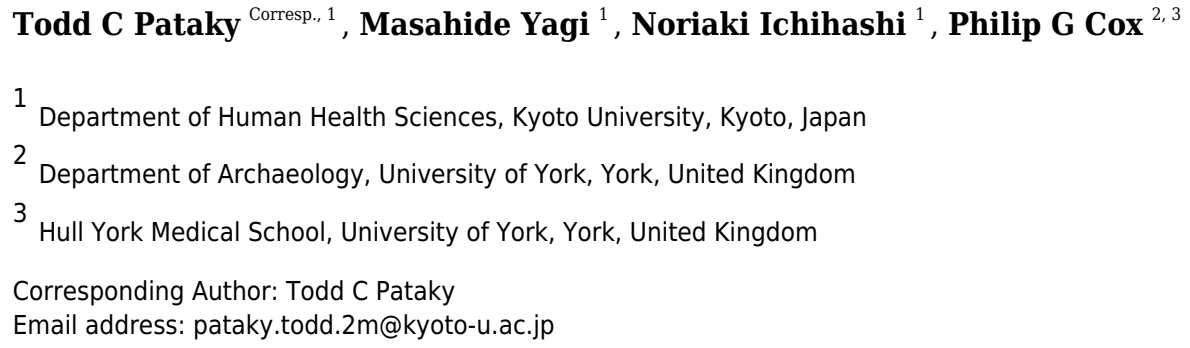

This paper proposes a computational framework for automated, landmark-free hypothesis testing of 2D contour shapes (i.e., shape outlines), and implements one realization of that framework. The proposed framework consists of point set registration, point correspondence determination, and parametric full-shape hypothesis testing. The results are calculated quickly $(<2 \mathrm{~s})$, yield morphologically rich detail in an easy-to-understand visualization, and are complimented by parametrically (or nonparametrically) calculated probability values. These probability values represent the likelihood that, in the absence of a true shape effect, smooth, random Gaussian shape changes would yield an effect as large as the observed one. This proposed framework nevertheless possesses a number of limitations, including sensitivity to algorithm parameters. As a number of algorithms and algorithm parameters could be substituted at each stage in the proposed data processing chain, sensitivity analysis would be necessary for robust statistical conclusions. In this paper, the proposed technique is applied to nine public datasets using a two-sample design, and an ANCOVA design is then applied to a synthetic dataset to demonstrate how the proposed method generalizes to the family of classical hypothesis tests. Extension to the analysis of 3D shapes is discussed. 


\title{
Landmark-free, parametric hypothesis tests regarding two-dimensional contour shapes using coherent point drift registration and statistical parametric mapping
}

\author{
Todd C. Pataky ${ }^{1}$, Masahide Yagi ${ }^{1}$, Noriaki Ichihashi ${ }^{1}$, and Philip G. Cox ${ }^{2,3}$ \\ ${ }^{1}$ Department of Human Health Sciences, Kyoto University Graduate School Of Medicine, \\ Kyoto, Japan \\ ${ }^{2}$ Department of Archaeology, University of York, York, UK \\ ${ }^{3}$ Hull York Medical School, University of York, York, UK \\ Corresponding author: \\ Todd C. Pataky ${ }^{1}$ \\ Email address: pataky.todd.2m@kyoto-u.ac.jp
}

\begin{abstract}
This paper proposes a computational framework for automated, landmark-free hypothesis testing of 2D contour shapes (i.e., shape outlines), and implements one realization of that framework. The proposed framework consists of point set registration, point correspondence determination, and parametric fullshape hypothesis testing. The results are calculated quickly $(<2 \mathrm{~s})$, yield morphologically rich detail in an easy-to-understand visualization, and are complimented by parametrically (or nonparametrically) calculated probability values. These probability values represent the likelihood that, in the absence of a true shape effect, smooth, random Gaussian shape changes would yield an effect as large as the observed one. This proposed framework nevertheless possesses a number of limitations, including sensitivity to algorithm parameters. As a number of algorithms and algorithm parameters could be substituted at each stage in the proposed data processing chain, sensitivity analysis would be necessary for robust statistical conclusions. In this paper, the proposed technique is applied to nine public datasets using a two-sample design, and an ANCOVA design is then applied to a synthetic dataset to demonstrate how the proposed method generalizes to the family of classical hypothesis tests. Extension to the analysis of 3D shapes is discussed.
\end{abstract}

\section{INTRODUCTION}

The statistical analysis of shape variation is relevant to a wide variety of academic fields including: evolutionary biology (Mitteroecker and Gunz, 2009), biomechanics (Pedoia et al., 2017), computer vision (Murphy-Chutorian and Trivedi, 2008), and many others (Da Costa and Cesar, 2000; Rohlf and Marcus, 1993; Adams et al., 2004, 2013). A key methodological framework for the statistical analysis of shape to have emerged in the literature is Geometric Morphometrics (Corti, 1993; Bookstein, 1996; Slice, 2007; Zelditch et al., 2012). Geometric Morphometrics consists of a variety of statistical techniques, ranging from classical hypothesis testing (e.g. Goodall, 1991) and classical dimensionality reduction techniques like principal component analysis (Adams et al., 2004) to machine learning techniques like unsupervised clustering (Renaud et al., 2005). This paper is concerned primarily with classical hypothesis testing as it pertains to shape analysis.

A common geometric morphometric approach to classical hypothesis testing regarding group differences (depicted in Fig.1a), consists of: (1) landmark definition, (2) spatial registration, and (3) Procrustes ANOVA (Goodall, 1991). Landmark definition refers to the manual identification and digitizing (i.e., XYZ coordinate specification) of homologous points on multiple objects, for example the corners on polyhedra. Spatial registration refers to the optimal, non-shearing affine alignment of a set of landmarks; that is, the optimal translation, rotation and scaling of each set of landmarks is calculated so that the 
the landmarks are optimally aligned in space. Procrustes ANOVA is effectively equivalent to classical ANOVA, where Procrustes distance is the dependent variable (Zelditch et al., 2012).

Landmarks with evolutionary, developmental or functional homology are essential for accurate interpretation of results (Hallgrimsson et al., 2015), especially for biological studies which seek to understand morphological variation in the context of evolution (e.g. Stayton, 2005; Morgan, 2009; Casanovas-Vilar and Van Dam, 2013; Dumont et al., 2016; Page and Cooper, 2017), ontogeny (e.g Klingenberg and McIntyre, 1998; Mitteroecker et al., 2004; Singleton, 2015) or function (e.g. Terhune et al., 2015; Toro-Ibacache et al., 2016). A key practical advantage of landmark approaches is that they impose problem tractability; they convert abstract, usually high-dimensional shape representations including images, scans and line contours, to a relatively small set of numeric coordinates which can be assembled into readily processable data formats like text files and spreadsheets. This practical advantage is reinforced by well-established statistical theory (e.g. Gower, 1975; Kendall, 1977, 1984, 1985; Kent, 1994; Rohlf, 1999) which describes a comprehensive solution for dealing with shape data's inherent dimensionality problem (Rohlf, 2000b,a; Collyer et al., 2015).

A common approach to landmark-based hypothesis testing is Procrustes ANOVA. While landmark data themselves are multivariate (i.e., multiple landmarks, each with multiple coordinates are used to describe a single shape), Procrustes ANOVA uses a univariate metric (Procrustes distance) to test shaperelevant hypotheses. One problem with this approach is that a single value is likely inadequate to fully characterize shape effects. Many other shape descriptors exist (Kurnianggoro et al., 2018), including both univariate metrics like eccentricity and multivariate metrics like geometric moments (Zhang and Lu, 2004). It has been argued that focus on relatively low dimensional shape metrics like these is necessary in order to achieve suitable statistical power, with the assumption that too many variables relative to the number of phenotypes can preclude hypothesis testing via parametric methods, especially for small samples (Collyer et al., 2015); one aim of this paper is to challenge that assertion, and to show that hypothesis testing is indeed possible for even high-dimensional representations of shape, and with suitably high statistical power for even relatively small sample sizes.

A related sample size-relevant theoretical limitation of Procrustes ANOVA is that there is no known parametric solution to the underlying Procrustes distance probability distributions. Consequently, statistical inference is conducted nonparametrically, often using bootstrapping or permutation techniques (Zelditch et al., 2012, pp.248-259). These nonparametric procedures are inherently poor for small sample sizes (Anderson and Braak, 2003; Brombin and Salmaso, 2009) because the probability distributions are constructed empirically and numerically, using the actual data, and both the precision and accuracy of these nonparametrically constructed distributions can decrease substantially with small sample sizes.

A variety of landmark-free or landmark-minimal methods also exist, including for example techniques that fit mathematical curves to shape outlines (Rohlf, 1990). One technique that has been particularly widely used is elliptical Fourier analysis (Claude, 2013; Bonhomme et al., 2014), which considers the spatial relations amongst neighboring points, and characterizes the spatial frequencies along the contour perimeter as a change-relevant representation of shape. Elliptical Fourier analysis has been frequently employed to analyse structures on which few homologous landmarks can be identified such as fins, jaws and teeth (e.g. Fu et al., 2016; Hill et al., 2018; Cullen and Marshall, 2019). These methods are highly relevant to the methods described in this paper, in that they deal with original, high-dimensional shape data like 2D contours and 3D surface scans.

While landmark-free or landmark-minimal methods initially operate on original high-dimensional shape data, they tend to use much lower-dimensional representations of shape when conducting classical hypothesis testing. For example, elliptical Fourier analysis tends to conduct hypothesis testing using a relatively small number (fewer than ten) harmonic coefficients (Bonhomme et al., 2014). Common landmark and landmark-free methods are thus similar from from a hypothesis testing perspective in that the hypothesis tests ultimately pertain to relatively low-dimensional shape metrics.

This main aim of this paper was to show that classical hypothesis testing is possible on original, highdimensional shape data, and in particular on continuous surfaces, without the need for low-dimensional shape representations, and with suitably high power even for analyses of relatively small samples. The methodology, which we refer to as 'continuous, mass-multivariate analysis' consists of a number of previously described techniques including: (1) point set registration, (2) correspondence, and (3) massmultivariate hypothesis testing. This combination of techniques allows one to conduct landmark-free hypothesis testing on original surface shapes. For interpretive convenience we limit focus to $2 \mathrm{D}$ contours 
(Bookstein, 1997; Carlier et al., 2016), but in the Discussion describe how the proposed methodology can be applied to 3D surfaces.

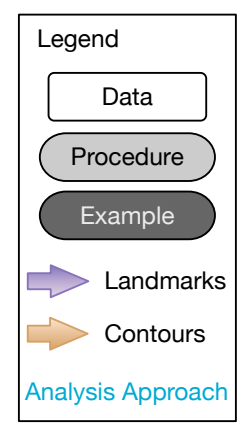

Analysis Approach

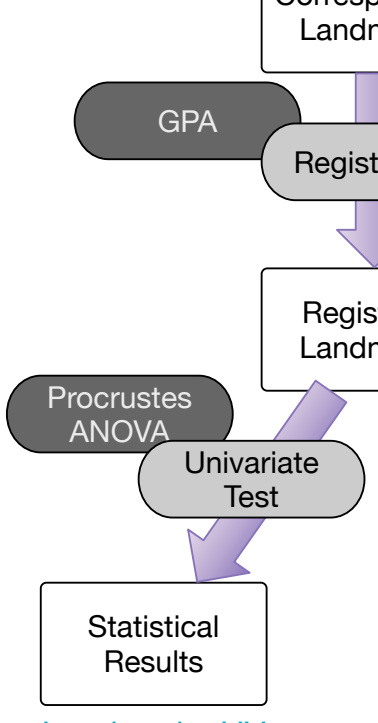

Landmarks UV

(a)

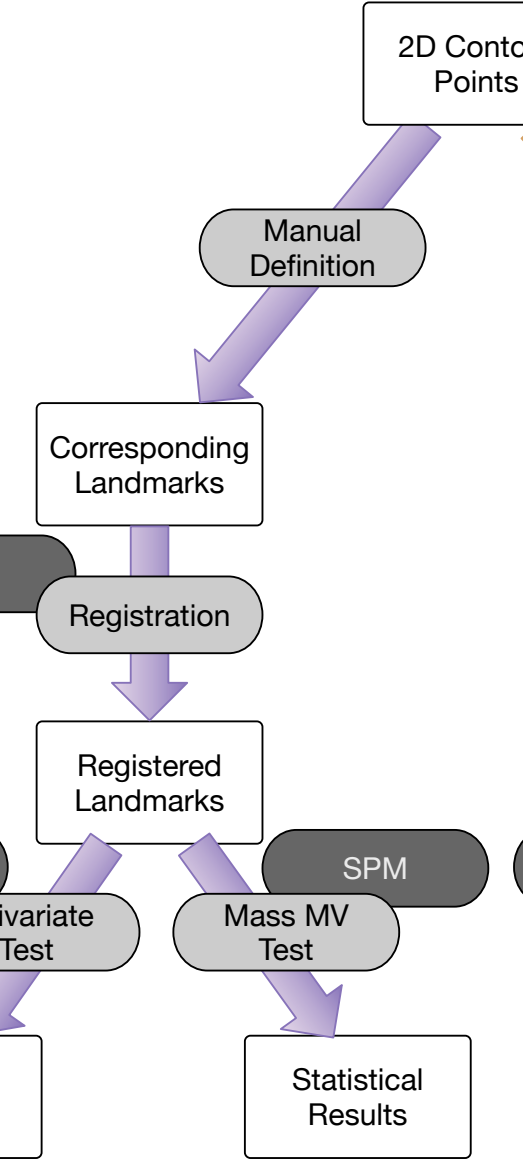

Landmarks Mass-MV

(b)

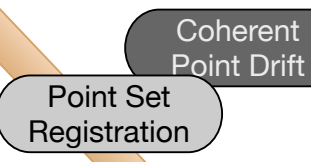

Registration

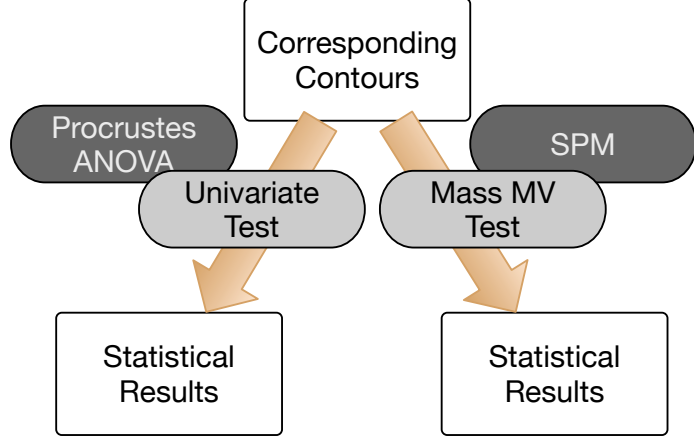

Contours UV

(c)
Contours Mass-MV

(d)

Figure 1. Overview of 2D contour data processing approaches employed in this paper. (a) The most common analysis approach, consisting of Generalized Procrustes Analysis (GPA) and Procrustes ANOVA for landmarks. (b) Same as (a), but using mass-multivariate (MV) analysis instead of Procrustes ANOVA's univariate (UV) approach. (c) and (d) are conceptually equivalent to (a) and (b), respectively, but operate on full contour data instead of landmark data, and can also be fully algorithmic. Statistical Parametric Mapping (SPM) is a methodology for mass-MV analysis of continuous data. See text for more details. 


\section{METHODS}

Analyses were conducted in Python 3.6.10 (van Rossum, 2019) using Anaconda 3.6.10 (Anaconda, 2020) and in R 3.6.2 (R Core Team, 2019) . Data processing scripts are available along with all original and processed data in this project's public repository at: https://github.com/0todd0000/ $\operatorname{lmfree} 2 \mathrm{~d}$.

\section{Datasets}

Nine datasets were analyzed (Fig.2). All datasets were taken from the the open-source 2D Shape Structure database (Carlier et al., 2016) (http://2dshapesstructure.github.io). The database consists of 70 different shape classes. Inclusion criteria for shape class were: (i) qualitatively similar geometry in at least 10 shapes (Fig.3), and (ii) at least four readily identifiable landmarks for all contour shapes.

Each dataset consisted of 20 contour shapes, where a 'dataset' represents a shape class (e.g., 'Bell' or 'Face') and individual shapes represent morphological variation within that shape class. We manually selected ten shapes from each dataset in a pseudo-random manner in order to span a range of effect sizes; in the Results, note that $\mathrm{p}$ values span a wide range $(p<0.001$ to $p>0.9)$. We selected just ten shapes primarily because it has been suggested that parametric procedures are unsuitable for the morphological analyses of small samples (Collyer et al., 2015), and we wished to demonstrate that the proposed parametric technique is indeed sufficiently powerful for small-sample analyses. Secondary reasons for considering just 10 shapes included: (1) qualitatively different within-class geometry, implying that statistical comparisons would be dubious if all 20 shapes were used, (2) inconsistent curvature characteristics (e.g., some with sharp corners, others with no discernible corners), implying landmarking difficulties, and (3) untrue contour data (e.g., internal loops and thus non-convex polygons) implying that contour parameterization was not possible for all shapes.

Two-sample tests were conducted on each dataset using the four approaches as described below. For replicability, the final set of ten shapes selected for analysis from each class are redistributed in this project's repository at: https://github.com/0todd0000/lmfree2d. Note that the ultimately selected contours had a variable number of contour points within each dataset (Table 1).

Table 1. Dataset count summary. Point counts refer to the original data from Carlier et al. (2016).

\begin{tabular}{|l|c|c|c|c|c|}
\hline Name & Shapes & \multicolumn{3}{|c|}{ Points } & Landmarks \\
\cline { 3 - 5 } & & Min & Median & Max & \\
\hline Bell & 10 & 101 & 104 & 185 & 8 \\
Comma & 10 & 101 & 104 & 108 & 4 \\
Device8 & 10 & 101 & 104 & 107 & 8 \\
Face & 10 & 103 & 104 & 106 & 4 \\
Flatfish & 10 & 100 & 102 & 112 & 5 \\
Hammer & 10 & 102 & 105 & 119 & 7 \\
Heart & 10 & 102 & 105 & 109 & 4 \\
Horseshoe & 10 & 106 & 109 & 128 & 6 \\
Key & 10 & 103 & 106 & 115 & 5 \\
\hline
\end{tabular}

\section{Data processing}

The 2D contour shape data were analyzed using four related approaches, consisting of the four combinations of (i) landmarks vs. contours, and (ii) univariate (UV) vs. mass-multivariate (mass-MV). These four approaches are summarized in Fig.1. The Landmarks-UV approach (Fig.1a) is common in the literature, none of the other approaches is common. The primary purpose of this study was to compare and contrast the Landmarks-UV and Contours-MassMV approaches (Fig.1a,d). We also employed intermediary approaches $($ Fig.1b,c) to more clearly highlight the differences between the two main approaches.

\section{Landmarks univariate (UV) analysis}

Landmarks were defined for each dataset as depicted in Fig.2. Both the number of landmarks (Table 1) and their locations were selected in an ad hoc manner, with the qualitative requirement of readily 
(a)

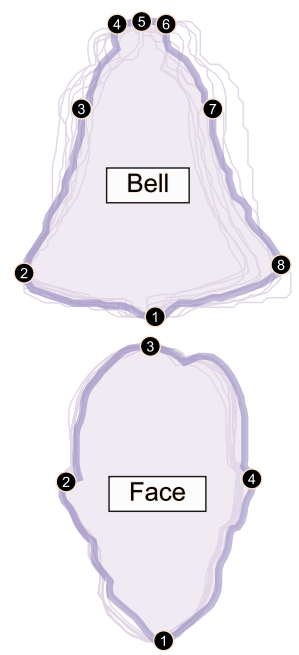

(g)

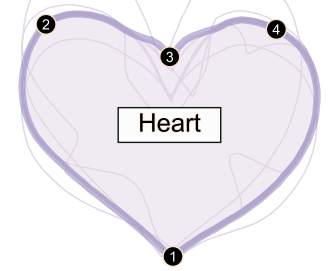

(b)

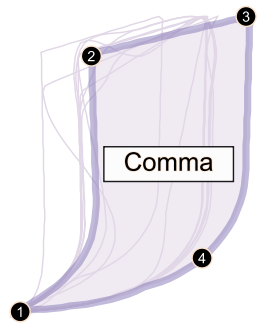

(e)

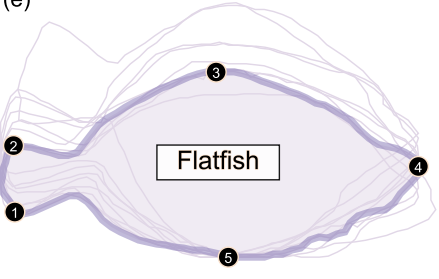

(h)

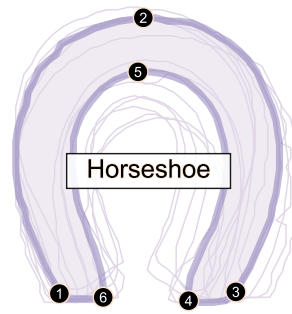

(c)

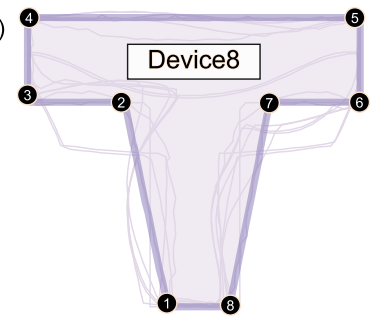

(f)

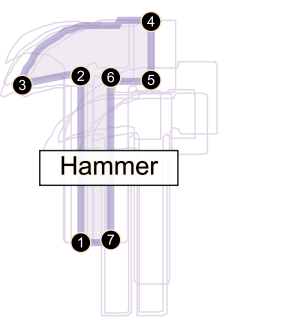

(i)

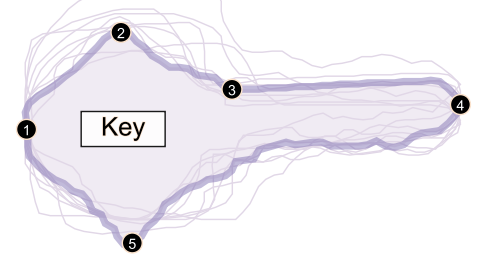

Figure 2. Overview of analyzed datasets. All contour data are available in the 2D Shape Structure Dataset (Carlier et al., 2016). For each dataset in this figure, one representative shape is highlighted, along with its numbered landmarks. Note that shape variance ranges from relatively small (e.g. Bell, Face) to relatively large (e.g. Device8, Heart).

(a) Cup

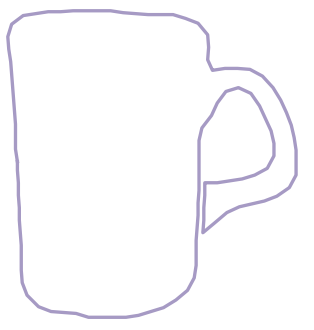

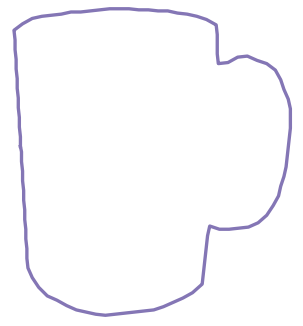

(b) Octopus
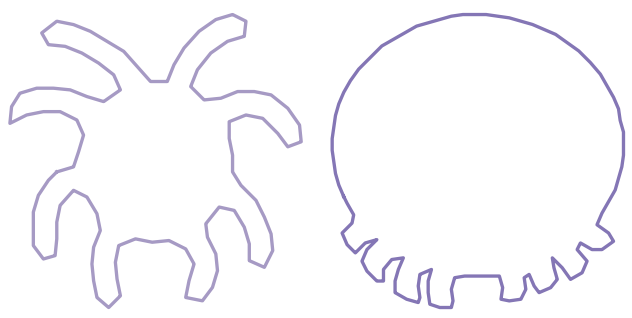

Figure 3. Shape class exclusion examples. Shape classes were excluded if they contained shapes with qualitatively different contour geometry. For example: (a) the 'cup' class was excluded because some shapes had unattached handles with holes and others had attached handles without holes. (b) The 'octopus' class was excluded because the eight appendages appeared in non-homologous locations.

identifiable, homologous locations. The ultimately selected landmarks arguably span a representative range of landmarking possibilities.

One operator used a mouse to manually digitize the landmarks for each of the 90 shapes (10 shapes for each of 9 datasets). The operator was ignorant of the final shape groupings for the ultimate two-sample tests (see below), implying that the landmarking was performed without grouping bias.

The landmarks were spatially registered using Generalized Procrustes Analysis (GPA) (Gower, 1975), and the resulting registered landmarks were analyzed in a univariate manner, using Procrustes ANOVA 
(Goodall, 1991) - a method which considers the variance in the Procrustes distance across a dataset. Note that the Procrustes distance is a scalar quantity that summarizes shape difference, and thus that this method is univariate. GPA and Procrustes ANOVA were both conducted using the geomorph package for R (Adams and Otárola-Castillo, 2013).

\section{Landmarks mass-multivariate (mass-MV) analysis}

This approach was identical to the Landmarks-UV approach described above, except for statistical analysis. The two-sample Hotelling's $T^{2}$ statistic was calculated for each landmark according to its definition:

$$
T_{i}^{2}=\frac{n_{1} n_{2}}{n_{1}+n_{2}}\left(\bar{r}_{1 i}-\bar{r}_{2 i}\right)^{\top} W_{i}^{-1}\left(\bar{r}_{1 i}-\bar{r}_{2 i}\right)
$$

where $i$ indexes landmarks, the subscripts " 1 " and " 2 " index the two groups, $n$ is sample size, $\bar{r}_{i}$ is the mean position vector of landmark $i$, and $W_{i}$ is the pooled covariance matrix for landmark $i$ :

$$
W_{i}=\frac{1}{n_{1}+n_{2}-2}\left(\sum_{j=1}^{n_{1}}\left(r_{1 i j}-\bar{r}_{1 i}\right)\left(r_{1 i j}-\bar{r}_{1 i}\right)^{\top}+\sum_{j=1}^{n_{2}}\left(r_{2 i j}-\bar{r}_{2 i}\right)\left(r_{2 i j}-\bar{r}_{2 i}\right)^{\top}\right)
$$

where the $i$ index is dropped for convenience in Eqn.2.

Statistical inference was conducted in a mass-multivariate manner, using Statistical Parametric Mapping (SPM) (Friston et al., 2007). SPM bases statistical inferences on the distribution of the maximum $T^{2}$ value $\left(T_{\max }^{2}\right)$, which can be roughly interpreted as the largest landmark effect, and which is defined as:

$$
T_{\max }^{2} \equiv \max _{i \in L} T_{i}^{2}
$$

where $L$ is the number of landmarks.

SPM provides a parametric solution to the distribution of $T_{\max }^{2}$ under the null hypothesis, so significance can be assessed by determining where in this distribution the observed $T_{\max }^{2}$ lies. Classical hypothesis testing involves the calculation of a critical threshold $\left(T^{2}\right)_{\text {critical }}$, defined as the $(1-\alpha)$ th percentile of this distribution, and all landmarks whose $T^{2}$ values exceed $\left(T^{2}\right)_{\text {critical }}$ are deemed significant at a Type I error rate of $\alpha$. This is a correction for multiple comparisons (i.e., across multiple landmarks) that is 'massmultivariate' in the following sense: 'mass' refers to a family of tests, in this case a family of landmarks, and 'multivariate' refers to a multivariate dependent variable, in this case is a two-component position vector. This is similar to traditional corrections for multiple comparisons like Bonferroni corrections, with one key exception: rather than using the total number of landmarks $L$ as the basis for the multiple comparisons correction, as the Bonferroni correction does, SPM instead solves the mass-MV problem by assessing the correlation amongst neighboring landmarks or semilandmarks, and using the estimated correlation to provide a less severe correction than the Bonferroni correction, unless there is no correlation, in which case the SPM and Bonferroni corrections are equivalent.

\section{Contours univariate (UV) analysis}

Similar to the Landmarks UV approach, this approach ultimately conducted Procrustes ANOVA, but did so on contour data rather than landmark data. This was achieved through two main processing steps: coherent point drift (CPD) point set registration (Fig.4) and optimum roll correspondence (Fig.5). Coherent point drift (CPD) (Myronenko and Song, 2010) is a point set registration algorithm that spatially aligns to sets of points that belong to the same or a similar object. Neither an equal number of points nor homologous points are required (Fig.4), making this approach useful for contours that have an arbitrary number of points.

Since contour points from arbitrary datasets may generally be unordered (Fig.5a), we started our analyses by randomly ordering all contour points, then applying CPD to the unordered points. We acknowledge that many $2 \mathrm{D}$ contour datasets consist of ordered points - including those in the database used for this study (Carlier et al., 2016) - but since 3D surface points are much more likely to be unordered, we regard unordered point support as necessary for showing that the proposed method is generalizable to $3 \mathrm{D}$ analyses. Following CPD, we re-ordered the points using parametric surface modeling 
(Bingol and Krishnamurthy, 2019), which fits a curved line to the contour, and parameterizes the contour using position $u$, where $u$ ranges from zero to one (Fig.6). This contour parameterization results in a continuous representation of the contour, from which an arbitrary number of ordered points (Fig.5b) can be used to discretize the contour of each shape for subsequent analysis. We used NURBS parameterization with B-spline interpolation (Bingol and Krishnamurthy, 2019) to calculate specific contour point locations. We then applied an optimum roll transformation, which found the value of $u$ for one contour that minimized the deformation energy across the two contours (Fig.5c,d).

We repeated contour parameterization, ordering, and optimum roll correspondence across all contour shapes, using the shape with the maximum number of contour points in each dataset as the template shape to which the nine other shapes were registered. Note that this registration procedure is unrelated to the traditional landmark analyses described in 'Landmark UV analysis' above, for which an equal number of points is a requirement of registration and analysis. The correspondence analysis step resulted in an equal number of contour points, upon which we conducted Procrustes ANOVA.

\section{Contours mass-multivariate (mass-MV) analysis}

This approach was identical to the Contours-UV approach, with the exception of statistical analysis, which we conducted using SPM as outlined above. Unlike the landmark data above, which are generally spatially disparate, contour points are spatially proximal, and neighboring points tend to displace in a correlated manner. For example, if one contour point in a specific shape lies above the mean point location, its immediate neighbors also tend to lie above the mean location). SPM leverages this correlation to reduce the severity of the multiple comparisons correction, and SPM solutions converge to a common $\left(T^{2}\right)_{\text {critical }}$ regardless of the number of contour points, provided the number of contour points is sufficiently large to embody the spatial frequencies of empirical interest, as outlined in classical signal processing theory (Nyquist, 1928).

As SPM uses parametric inference to calculate the critical $T^{2}$ threshold, and Procrustes ANOVA uses nonparametric inference, we also conduct Contours Mass-MV analysis using statistical non-parametric mapping (Nichols and Holmes, 2002), which uses permutation to numerically build the $T_{\max }^{2}$ distribution under the null hypothesis. This permutation approach converges to the parametric solution when the residuals are normally distributed (i.e., point location variance follows an approximately bivariate Gaussian distribution). All SPM analyses were conducted in spm1d (Pataky, 2012); note that one-dimensional SPM is sufficient because the contour domain $(U)$ is one-dimensional (Fig.6).

(a) Original

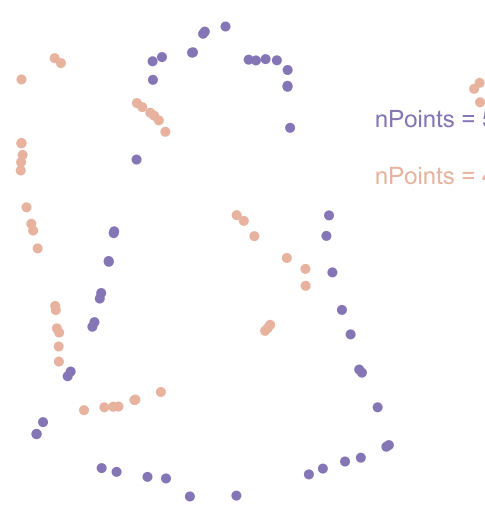

(b) CPD-registered

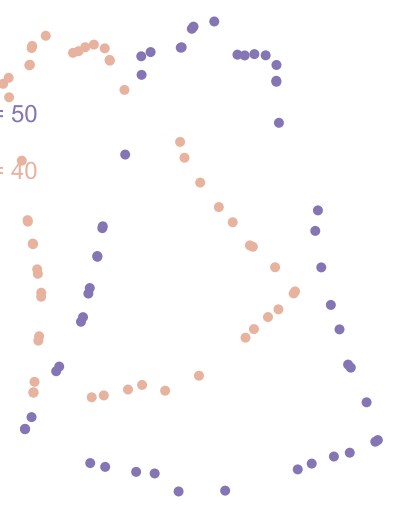

Figure 4. Example point set registration using the coherent point drift (CPD) algorithm (Myronenko and Song, 2010). Note that CPD requires neither corresponding points, nor an equal number of points. 
(a)

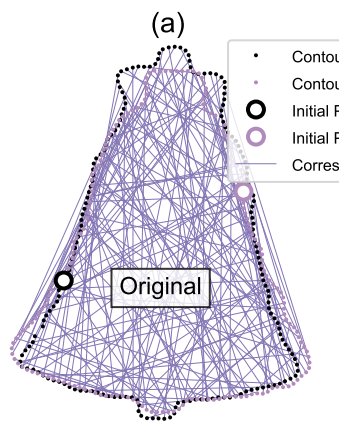

(b)

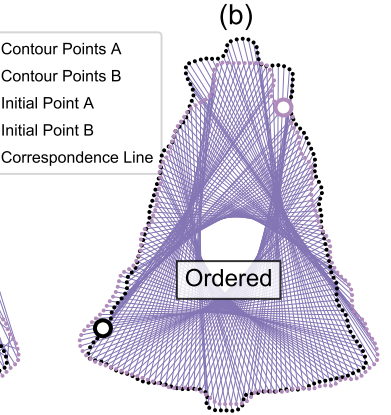

(c)

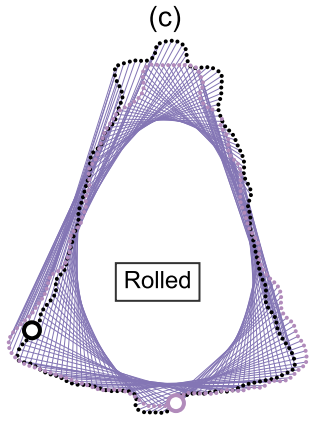

(d)

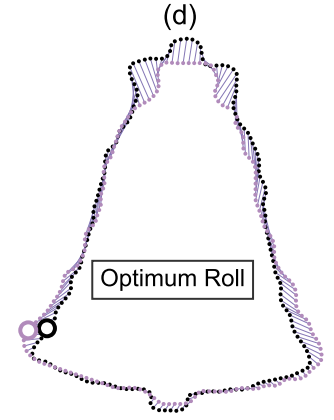

Figure 5. Example optimum roll correspondence. (a) Original data, consisting of an equal number of contour points, arranged in a random order. (b) Ordered points; clockwise along the contour. (c) Rolled points; moving the initial point of contour B brings the shapes into better correspondence. (d) Optimally rolled points; the total deformation energy across all points (i.e. the sum-of-squared correspondence line lengths) is minimum.

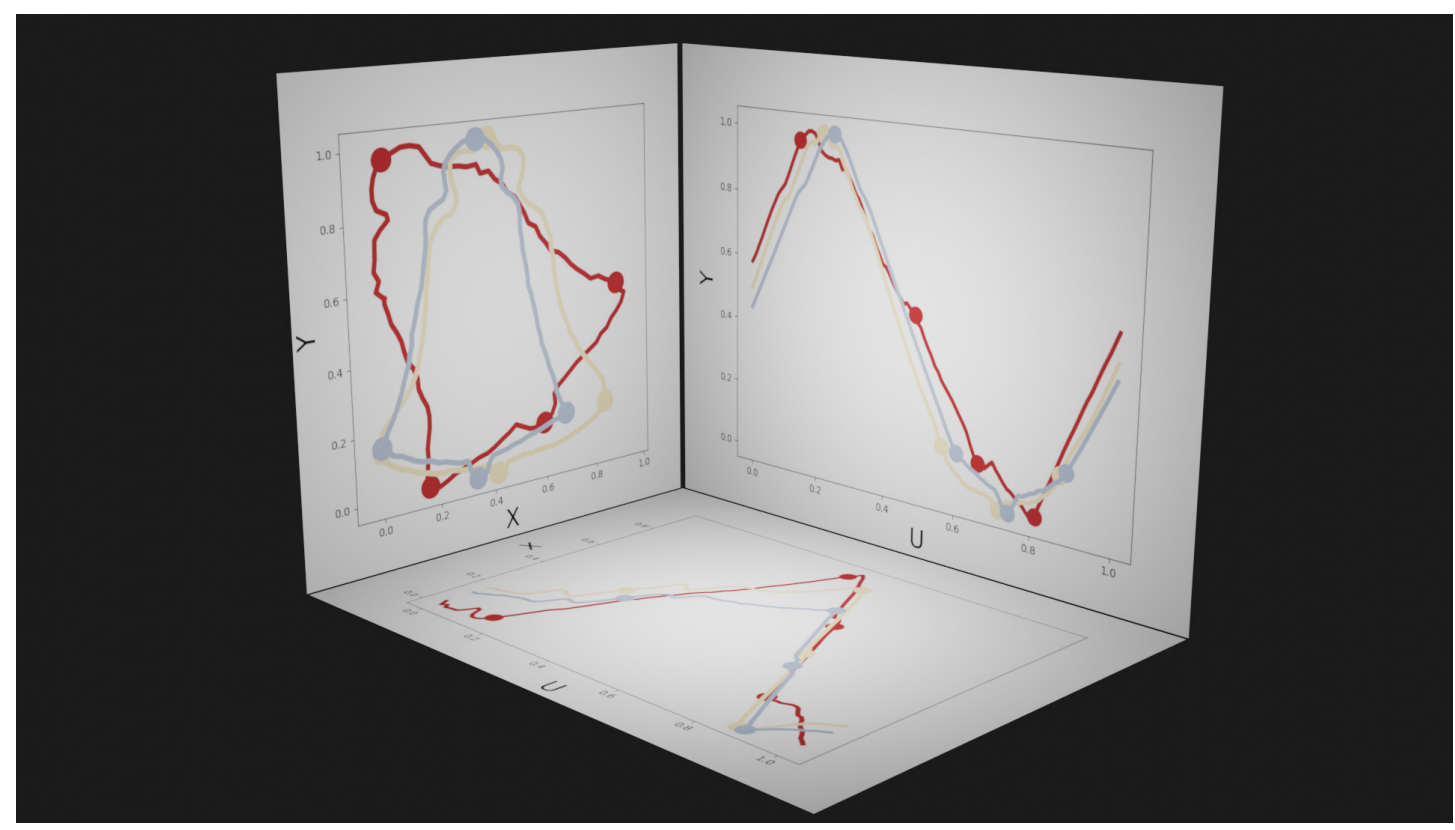

Figure 6. Example parametric representations of 2D contour shape. Dots represent manually defined landmarks, and are shown as visual references. Left panel (XY plane): the spatial plane in which shape data are conventionally presented. The three colors represent different shapes. Bottom panel (UX plane) and right panel (UY plane): abstract planes in which $U$ represents the parametric position (from 0 to 1 ) along the contour; positions $\mathrm{U}=0$ and $\mathrm{U}=1$ are equivalent. 
Table 2. Statistical results summary, probability values. As nonparametric inference yielded similar $p$ values (see Results), only parametric $\mathrm{p}$ values are reported in this table for brevity.

\begin{tabular}{|l|c|c|c|c|}
\hline \multirow{2}{*}{ Name } & \multicolumn{2}{|c|}{ Landmarks } & \multicolumn{2}{c|}{ Contours } \\
\cline { 2 - 5 } & UV & Mass-MV & UV & Mass-MV \\
\hline Bell & 0.130 & 0.302 & 0.084 & $\mathbf{0 . 0 4 1}$ \\
Comma & 0.155 & 0.294 & 0.719 & 0.327 \\
Device8 & $\mathbf{0 . 0 2 2}$ & 0.214 & 0.433 & 0.681 \\
Face & $\mathbf{0 . 0 2 5}$ & 0.103 & 0.052 & $\mathbf{0 . 0 1 3}$ \\
Flatfish & $\mathbf{0 . 0 2 3}$ & $\mathbf{0 . 0 1 6}$ & $\mathbf{0 . 0 2 6}$ & $\mathbf{0 . 0 0 1}$ \\
Hammer & 0.708 & 0.206 & 0.417 & $<\mathbf{0 . 0 0 1}$ \\
Heart & 0.940 & 0.976 & 0.544 & $\mathbf{0 . 0 1 6}$ \\
Horseshoe & 0.084 & $\mathbf{0 . 0 0 8}$ & $\mathbf{0 . 0 0 6}$ & $\mathbf{0 . 0 0 1}$ \\
Key & 0.532 & 0.270 & $\mathbf{0 . 0 1 3}$ & $\mathbf{0 . 0 2 2}$ \\
\hline
\end{tabular}



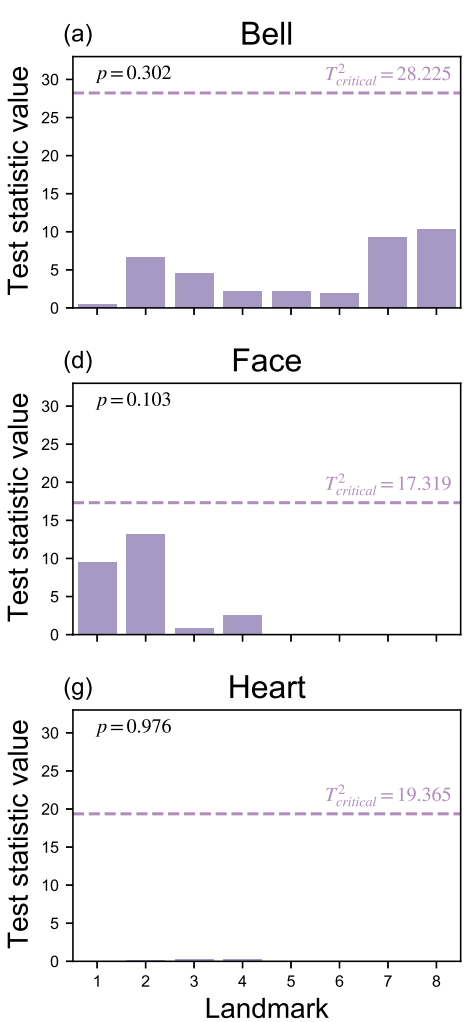
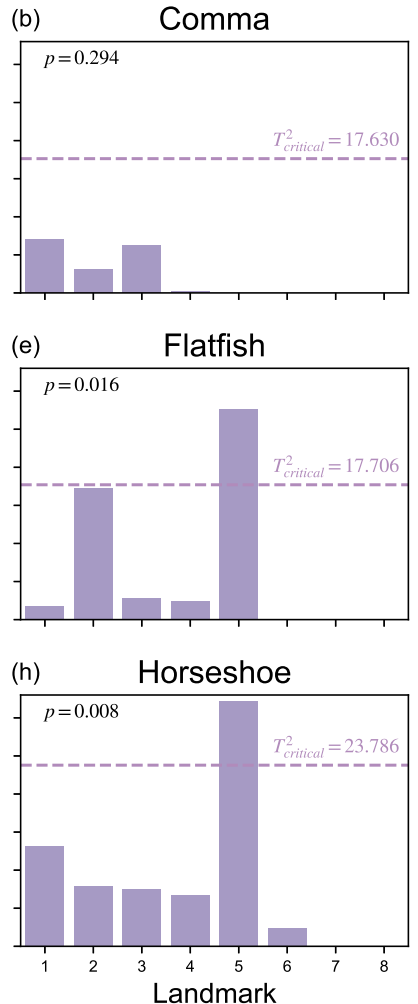
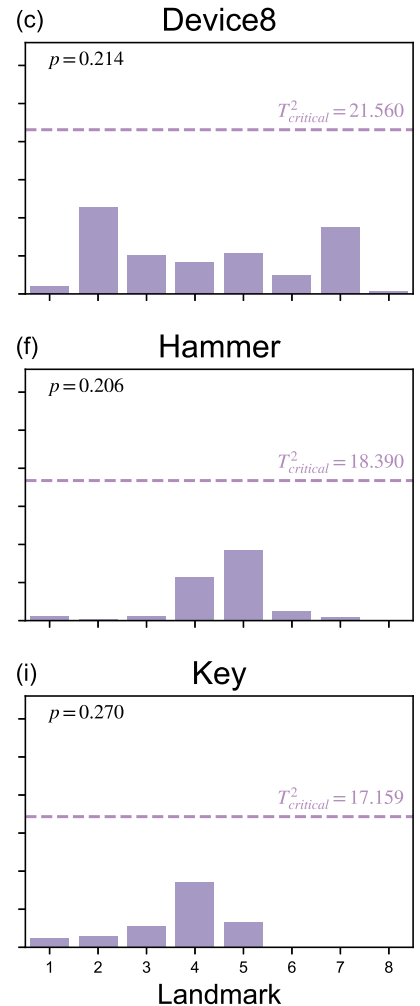

Figure 7. Landmark results from mass-multivariate testing. Landmark-specific $T^{2}$ values are presented along with the critical threshold at $\alpha=0.05$, and probability values for the overall mass-multivariate test.

Table 3. Execution durations (unit: ms). Averages across the nine datasets. Procrustes ANOVA (Proc-ANOVA) involved 1000 iterations for each dataset. Average SnPM durations (not shown in this table) were 344.0 and $6336.0 \mathrm{~ms}$ for Landmarks Mass-MV and Contours Mass-MV, respectively.

\begin{tabular}{|l|l|c|c|c|c|}
\hline Category & \multirow{2}{*}{ Procedure } & \multicolumn{2}{|c|}{ Landmarks } & \multicolumn{2}{c|}{ Contours } \\
\cline { 3 - 6 } & & UV & Mass-MV & UV & Mass-MV \\
\hline \multirow{4}{*}{ Registration } & CPD & - & - & 414.1 & 414.1 \\
& Point Ordering & - & - & 327.9 & 327.9 \\
& Interpolation & - & - & 835.1 & 835.1 \\
& Correspondence & - & - & 40.9 & 40.9 \\
& GPA & 6.7 & 6.7 & 8.5 & - \\
\hline \multirow{2}{*}{ Hypothesis test } & Proc-ANOVA & 60.0 & - & 99.0 & - \\
& SPM & - & 39.3 & - & 66.8 \\
\hline Total & & 66.7 & 46.0 & 1725.5 & 1684.8 \\
\hline
\end{tabular}



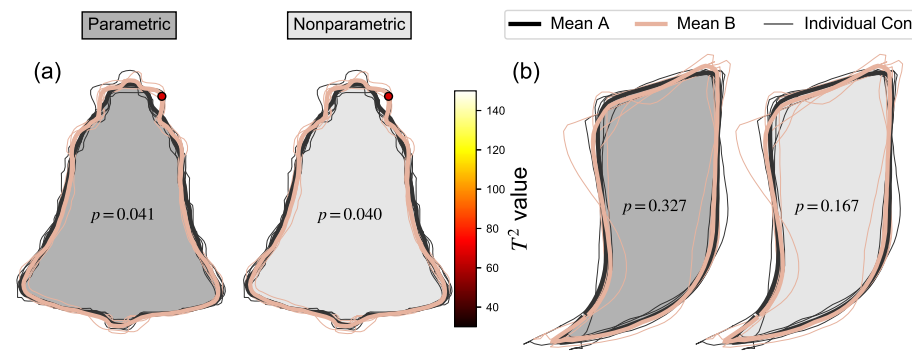

Significant Contour Points (at $\alpha=0.05$ )
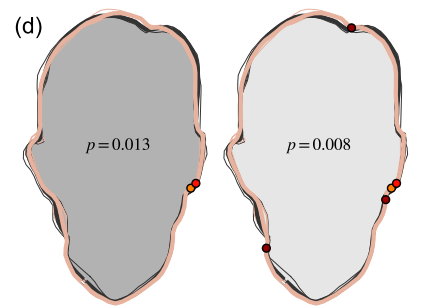

(e)

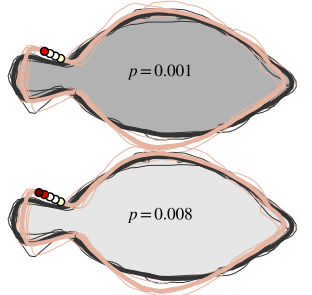

(c)

(g)

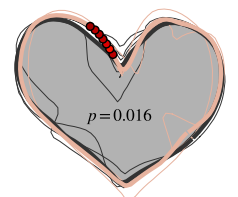

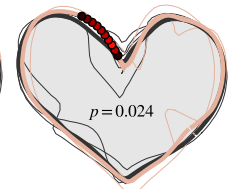
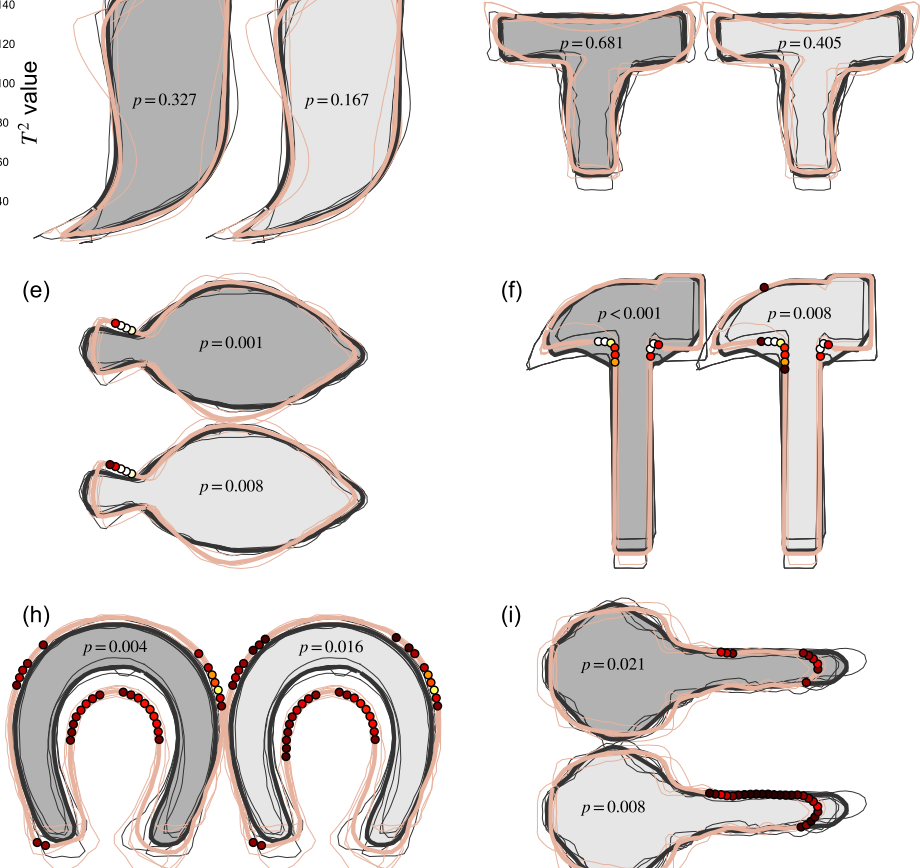

(f)

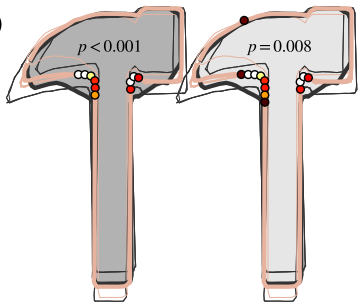

(i)

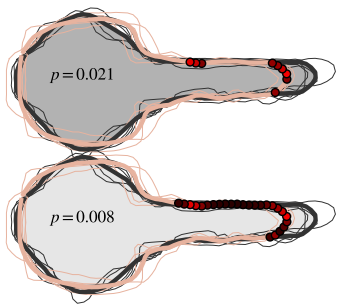

Figure 8. Contours mass-multivariate results using Statistical Parametric Mapping (SPM). Results for both parametric and nonparametric inference are shown. $\mathrm{P}$ values represent the probability that random variation in the Mean A contour would produce a deformation as large as in the observed Mean B, given the estimated contour variance. Dots on the Mean B contour represent contour points whose $T^{2}$ values exceeded the threshold for significance at $\alpha=0.05$; if the maximum $T^{2}$ value did not reach this threshold, the $\mathrm{p}$ value is greater than $\alpha$, and no dots are shown. 
243

244

\section{DISCUSSION}

\section{Main findings}

This study's main result is the demonstration that it is possible to conduct fully automated, landmark-free, parametric hypothesis testing regarding whole $2 \mathrm{D}$ contour shapes, irrespective of the number of points and point ordering in the original contour data. These analyses can be executed relatively quickly; the current non-optimized implementation required less than $2 \mathrm{~s}$ for all analysis steps (Table 3 ). The proposed analysis framework (Fig.1d) consists of families of previous techniques including: point set registration (e.g. Myronenko and Song, 2010), point correspondence algorithms (e.g. Loy et al., 2000; Myronenko and Song, 2010), and mass-multivariate testing (Friston et al., 2007; Taylor and Worsley, 2008; Chung et al., 2010), and some of these techniques have been used for classical hypothesis testing regarding shapes in the past (Taylor and Worsley, 2008; Chung et al., 2010). A variety of landmark-free techniques have also been previously proposed (e.g. Wuhrer et al., 2011; Taylor and Worsley, 2008; Chung et al., 2010) Nevertheless, these techniques have not, to our knowledge, been previously combined into a general hypothesis testing framework - from raw data to statistical results - as depicted in Fig.1d. The main novelty of this paper is thus the demonstration that it is possible to fully automate data processing from raw 2D contour data to final hypothesis testing results.

The second main novelty of this paper is the demonstration that parametric hypothesis testing is possible when conducted at the whole-contour level. We stress that 'possible' implies neither 'valid' nor 'appropriate'; demonstrating the validity and appropriateness of the proposed method would require substantial empirical efforts over a range of datasets, data modalities, experimental designs, and applications, in addition likely to simulation studies, and as such assessing validity and appropriateness are beyond the scope of this paper. We also stress that 'possible' does not imply that one should use the proposed technique in isolation. We believe that the proposed technique offers unique information that is complimentary to other techniques, and that ideally the results of multiple analysis techniques should be corroborated to build interpretive robustness.

The proposed analysis framework (Fig.1d) offers various improvements over landmark analysis (Fig.1a) including: (1) the modeling flexibility of classical hypothesis testing, (2) increased objectivity due to avoidance of subjective landmark definition and selection, (3) increased speed due to avoidance of manual work, and (4) unique, implicit morphological meaning in hypothesis testing results. We acknowledge that each of these improvements also involve limitations, and we address these limitations below. We stress that 'objectivity' implies none of 'accurate', 'useful' or 'interpretable'. We use 'objective' instead primarily to mean 'algorithmic'.

\section{Statistical Parametric Mapping (SPM)}

SPM, like most parametric tests, assumes normality, so in this case SPM assumes that the spatial variability of all contour points are distributed in a bivariate Gaussian manner. This distributional assumption could be directly tested using distributional tests in a point-by-point manner. In this paper, instead of directly testing for distributional adherence, we instead tested the assumption indirectly, by conducting nonparametric tests (Fig.8), which do not assume bivariate normality. In this case there were minor quantitative differences between the parametric and nonparametric results, but overall the qualitative interpretations were largely unaffected by the use of parametric vs. nonparametric analysis. This represents relatively strong (albeit indirect) evidence that the parametric approach's distributional assumptions are appropriate at best, or largely inconsequential at worst, for these particular datasets. This however does not imply that parametric inference is appropriate for all datasets, so distributional assumptions should generally be tested for all datasets, possibly indirectly through nonparametric tests like those conducted in this paper.

Although this paper considered only two-sample tests, SPM supports all classical hypothesis testing procedures, ranging from simple linear regression to MANCOVA (Friston et al., 2007), thereby making the proposed framework highly flexible to arbitrary experimental designs. To emphasize this point, and how it may be valuable for general shape analysis, we conducted a set of supplementary analyses using synthetic data involving simple, circular shapes with controlled morphological effects (Fig.9a,b). The controlled effects included a size-dependent signal, which was modeled using a Gaussian contour pulse that increased in amplitude with increasing shape size (as defined by the shape's average radius) (Fig.9a), and a group-dependent signal, which was modeled similarly, but which was applied to just one of two hypothetical groups (Fig.9b). To isolate and emphasize design flexibility, and to eliminate registration and correspondence as potential sources of error, we controlled both by sampling at 101 evenly distributed 
angular displacements with respect to the horizontal axis. We considered two MANCOVA possibilities: analysis of the original, unscaled dataset (Fig.9a), and analysis of the scaled / registered dataset (Fig.9b). We applied a single MANCOVA model, which modeled both shape size (i.e., mean shape radius) and group, and which thereby afforded consideration of both (1) size effects, with group effects linearly removed, and (2) group effects, with size effects linearly removed. Size effects for the original, unscaled data naturally showed very large test statistic values at all contour points (Fig.9c). In contrast, size effects for the registered data correctly isolated the modeled size-dependent signal (Fig.9d). Group effects were practically identical for both the original, unscaled data and the registered data (Fig.9e,f), emphasizing the point that MANCOVA can be used to remove size-related effects in lieu of registration. More generally, this analysis shows that the proposed framework is highly flexible, and can be used with arbitrary continuous and categorical independent variables, provided these variables adhere to the requirements of classical linear design modeling. We nevertheless caution readers that the (Fig.9) analyses consider close-to-ideal data, for which registration and correspondence are near-perfectly controlled. For real dataset analysis, both registration and correspondence generally introduce errors that may or not affect the ultimate hypothesis testing results. Results' sensitivity to data processing algorithms and their parameters must be considered in general analyses.

\section{Comparison with landmarking and other methods}

The proposed methodology partially overcomes limitations of landmark selection, and the corresponding susceptibility to bias (Arnqvist and Martensson, 1998; Rohlf, 2003; Fruciano, 2016); shape-to-shape landmark identification is often manual and therefore subjective. Algorithmic landmark identification is nevertheless possible (Claes et al., 2011; Strait and Kurtek, 2016), and indeed modern machine learning techniques have been shown to substantially improve landmark detection, with the promise of eliminating landmark-associated subjectivity (Morris, 2003; Young and Maga, 2015; Strait and Kurtek, 2016; Devine et al., 2020). Like automated landmarking, the proposed method can be used with little-to-no subjective intervention, implying generally more repeatable results. Here 'objective' does not necessarily mean 'accurate' or 'appropriate'; it simply means that results are expected to be more reproducible than the results from more subjective methods. Determining the accuracy and appropriateness of all methods, including the proposed one, requires substantial empirical effort across a range of data modalities and applications.

We also note that the proposed landmark-free approach is just one end of the spectrum, where manual landmark definition is the other, and that a variety of alternative techniques occupy positions between these two extremes. For example, semilandmarks (Mitteroecker and Gunz, 2009) provide an objective way to fill spatial gaps between landmarks, thereby creating a continuous surface. From the perspective of the proposed method, semilandmarks represent the results of piecewise registration over the domain $u$ (Fig.6), or equivalently a hybrid registration method consisting of both algorithmic and manual components (Ramsay and $\mathrm{Li}, 1998$ ). As there are a plethora of automated techniques for geometrical matching (Holden, 2008), the proposed framework regards these techniques each as objective, substitutable, yet each imperfect components, whose assumptions and parameters could ultimately affect the final results. From this perspective, a second layer of objectivity could be added to the proposed framework, whereby different techniques and/or parameters are iteratively substituted in a sensitivity framework, to objectively discern the numerical stability of the final results, as well as the boundaries of that stability (Pataky et al., 2014).

Landmarks and other low-dimensionality representations of shape - including harmonic coefficients from elliptic Fourier analysis (Bonhomme et al., 2014) — embody a second important limitation: a potentially over-simplified representation of shape. In the case of landmarks, a danger of over-simplification arises from the Nyquist theorem: under-sampling a continuous process (including the continuous spatial surface of an object) can lead to aliasing, whereby the under-sampled measurement can misrepresent the true characteristics of the underlying object (Nyquist, 1928), and can even reverse statistical interpretations through mechanisms such as regional conflation (Pataky et al., 2008). This latter problem of shape simplification can nevertheless be solved by the use of semi-landmarks (Bookstein, 1997; Adams et al., 2004) which, as argued above, can be regarded as a specific approach to shape registration, implying that semi-landmark approaches could interface easily with the proposed technique.

An advantage of the proposed method is processing speed. The current, non-optimized analyses executed in under $2 \mathrm{~s}$, with statistical inference itself requiring well under $100 \mathrm{~ms}$ (Table 3). We 

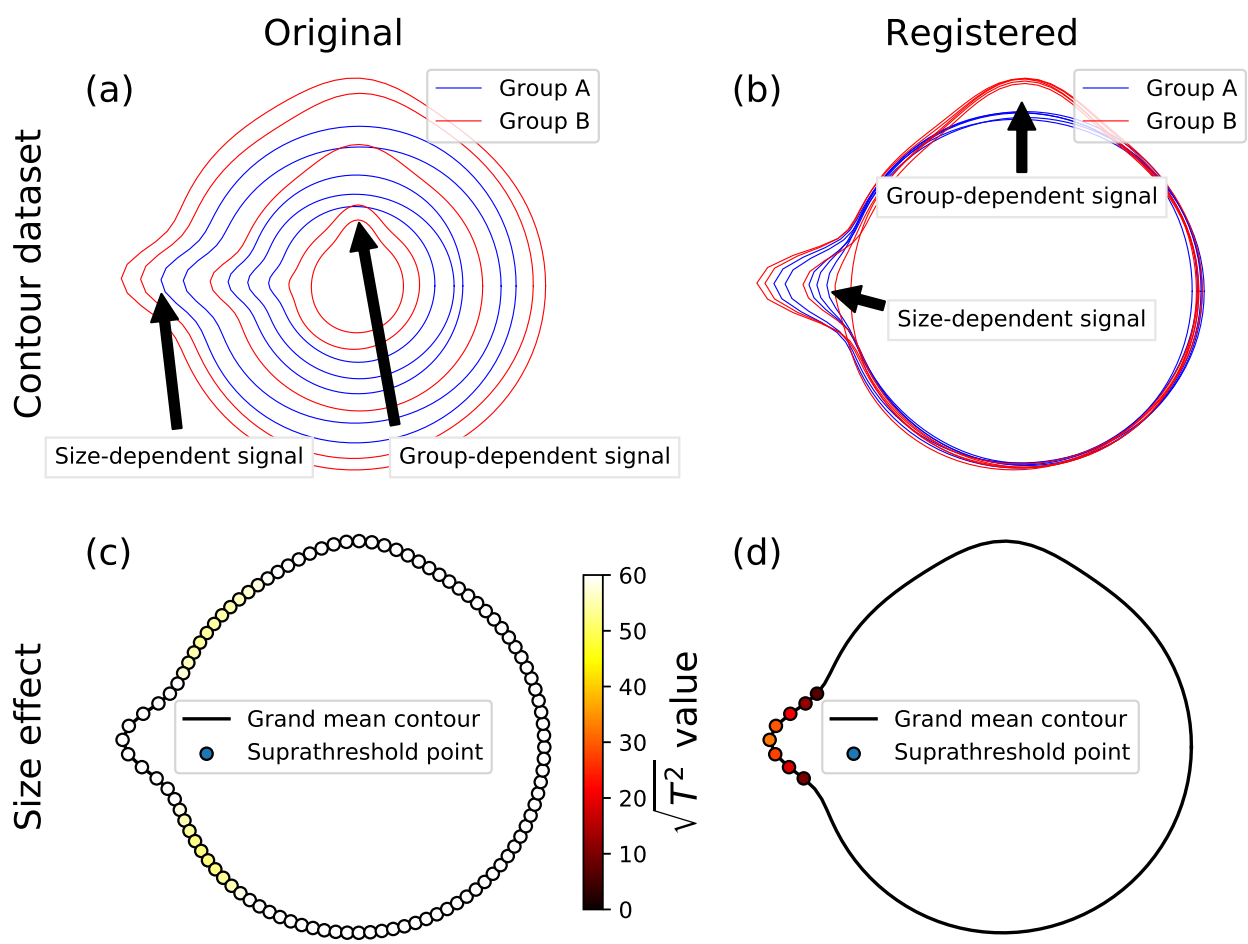

(d)
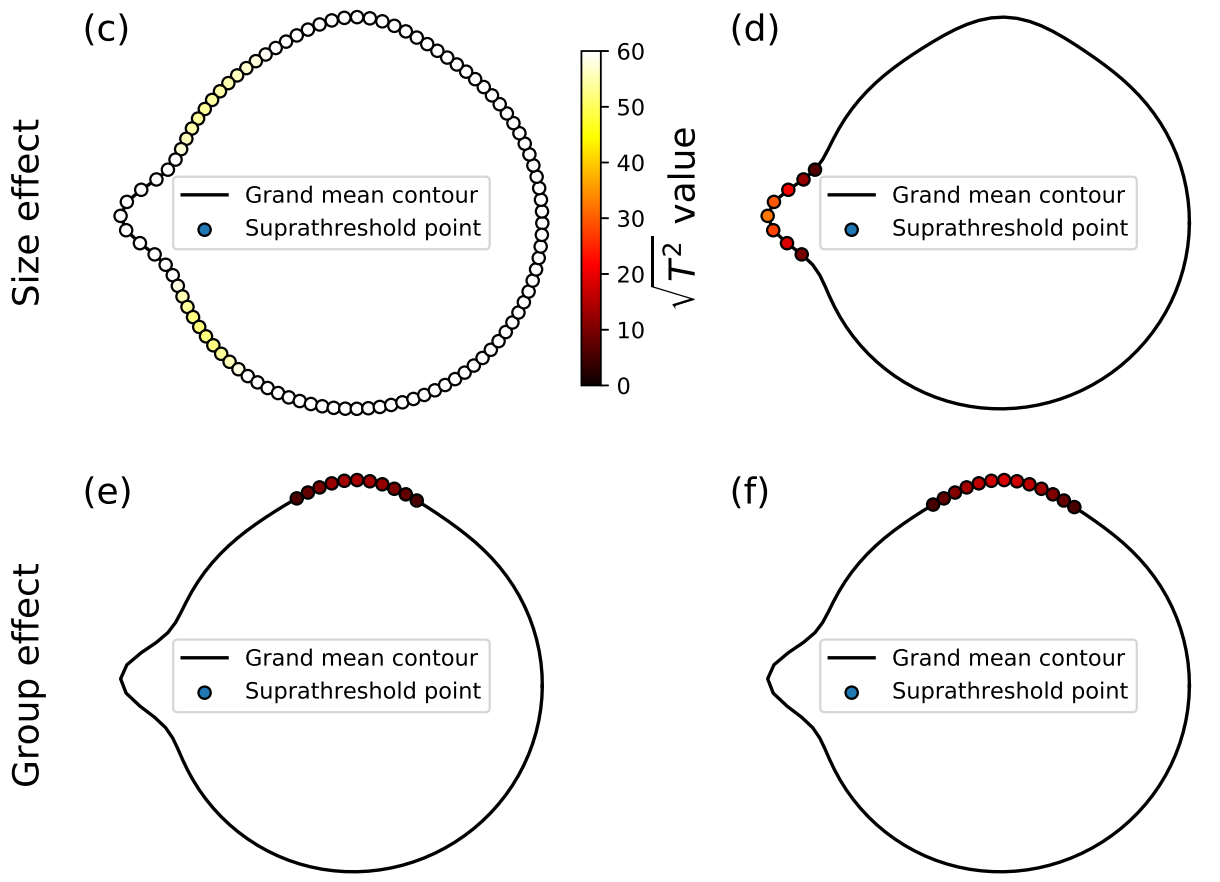

(f)

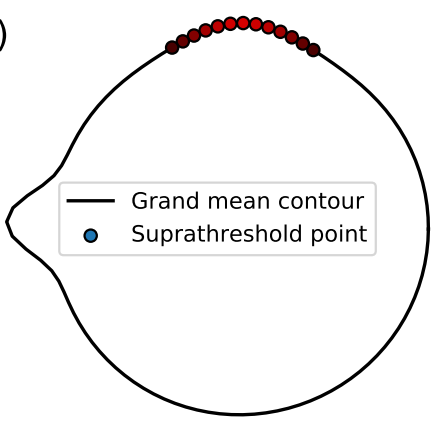

Figure 9. Example MANCOVA using synthetic data; for simplicity, data were generated to have (i) a relatively large signal:noise ratio, and (ii) close-to-perfect correspondence, by sampling at 101 equally spaced angular distances around the contour. (a) The original contour dataset, consisting of five noisy circles for each of two groups, with systematically different mean radii, and also with both group- and size-dependent signal, where 'size' was considered to be the mean radius, and where 'signal' implies true morphological difference. Note that the size-dependent signal is more easily perceived in panel (a), and that the group-dependent signal is more easily perceived in the next panel. (b) Registered contours. (c,d) Size effects from MANCOVA for the original and registered data; the test statistic is presented as $\sqrt{T^{2}}$ because a linear $T^{2}$ scale would result in imperceivable color differences (i.e., the panel (c) points would be all white, and the points in the other panels would all be close-to-black). (e,f) Group effects from MANCOVA for the original and registered data; note that the (e) and (f) results are similar because MANCOVA accounts for size-related effects in the 'Original' data.

acknowledge that other data processing steps, including image segmentation and registration for example, can require substantial effort, so we caution readers that the reported execution speeds do not necessarily translate to reduced laboratory hours. The primary advantage in our view is instead the promotion of sensitivity analysis: since the entire data processing chain can be executed relatively rapidly, it would be possible to systematically adjust algorithm parameters, and even swap algorithms, in a sensitivity loop, to probe the robustness of particular results. 
Another advantage of the proposed method is implicit morphological information. The proposed method yields results that are rich in morphological detail (Fig.8) which, much like a highlighted photograph or x-ray image, can be readily interpreted at a glance. Since SPM operates directly on (registered) contours, without reducing the object-of-hypothesis-testing to a single abstract metric (like Procrustes ANOVA), or to a small handful of abstract metrics (like elliptical Fourier analysis), SPM results embody morphological meaning insofar as contours themselves embody morphological meaning. While individual contour points do not necessarily embody meaning, one could argue that the set of all contour points collectively embodies substantial morphological meaning. This perspective is analogous to a pixel-and-image argument. The color of a single pixel is largely irrelevant to the overall interpretation and meaning of an image. Similarly, the test statistic value at a single contour point is itself largely irrelevant to the overall morphological interpretation of SPM results; morphological meaning is instead encapsulated implicitly in the overall excursion set, where 'excursion set' means the set of supra-threshold contour points, like those in Fig.8. Regardless of the quality of morphological meaning, SPM results must be viewed as just one set of results, which may or may not embody useful morphological information, and which should be considered along with other, more explicit morphological methods like Procrustes ANOVA and elliptical Fourier analysis.

Considering last specific results from this paper, a particularly unintuitive set of results was observed for the Device8 dataset, for which UV analysis yielded the smallest $\mathrm{p}$ value (0.022), and for which no other method yielded significance $(p>0.2)$ (Table 2$)$. This result was likely caused by widespread but relatively small-magnitude mean-shape differences (Fig.8c); since the deformation is widespread it would be detected by a general deformation metric like Procrustes distance, but since the deformation magnitude is relatively small it would not be detected by local contour-point methods like SPM. The interpretation is emphasized in the Flatfish dataset, where general deformations were similarly broadly distributed across the contour, but maximal local deformations were greater (Fig.8e), which yielded significance in all methods (Table 2). Nevertheless, this interpretation appears to be inconsistent with the Horseshoe dataset, which exhibited both large and widely distributed deformation (Fig.8h), but which also failed to yield significant UV results (Table 2). Nevertheless, this apparent consistency may be resolved by considering the large variability in the Horseshoe dataset, particularly at the selected landmarks (Fig.2h). To more completely resolve such apparent inconsistencies, and more generally to understand the nature of landmark- vs. contour-based methods, it would be necessary to consider individual contour points, their deformations, and their covariances.

\section{Generalization to 3D analysis}

While this paper was limited to 2D analysis, it should be noted that the proposed analysis framework (Fig.1d) can be readily extendable to the morphological analysis of 3D surfaces. Similar to the unwrapping of 2D contours onto a 1D domain $u$ (Fig.6), 3D surfaces can be unwrapped onto a 2D domain $u v$ Fig.10, and methods like SPM (Friston et al., 2007) can be used to conduct domain-level hypothesis testing regarding these unwrapped data. This domain-wide testing is possible due to the underlying model of domain-level variance, which SPM models as smooth, Gaussian random fields, and which can be extended to arbitrarily high-dimensional domains with arbitrary geometry (Adler and Taylor, 2007). For the current paper involving 2D shapes, the (flattened) domain is one-dimensional, and the dependent variable is a two-component position vector; that is, a two-component position is defined at all locations $u$ along the contour. Similarly, for 3D surfaces, the (flattened) domain is two-dimensional and the dependent variable is a three-component position vector, where position is defined at all locations $u v$ across the surface. A variety of computational tools exist for 3D geometry flattening (e.g. Dale et al., 1999; Sawhney and Crane, 2017), so 3D implementations of the proposed method could presumably proceed in a fully automated manner.

\section{Limitations}

The proposed mass-multivariate framework (Fig.1d) has a number of limitations. The most severe of these is sensitivity to algorithmic specifics. For example, simply by randomly changing the order of the points, it is possible to yield qualitatively different results (Fig.11). Systematic, random variations of point ordering would be necessary for assessment of the results' sensitivity, but in our view this would be insufficient because ultimate results may also be sensitive to other particulars including, for example, specific parameter values used in contour parameterization, registration, and correspondence algorithms. In other words, one should regard the results as potentially sensitive to all data processing steps, and not 

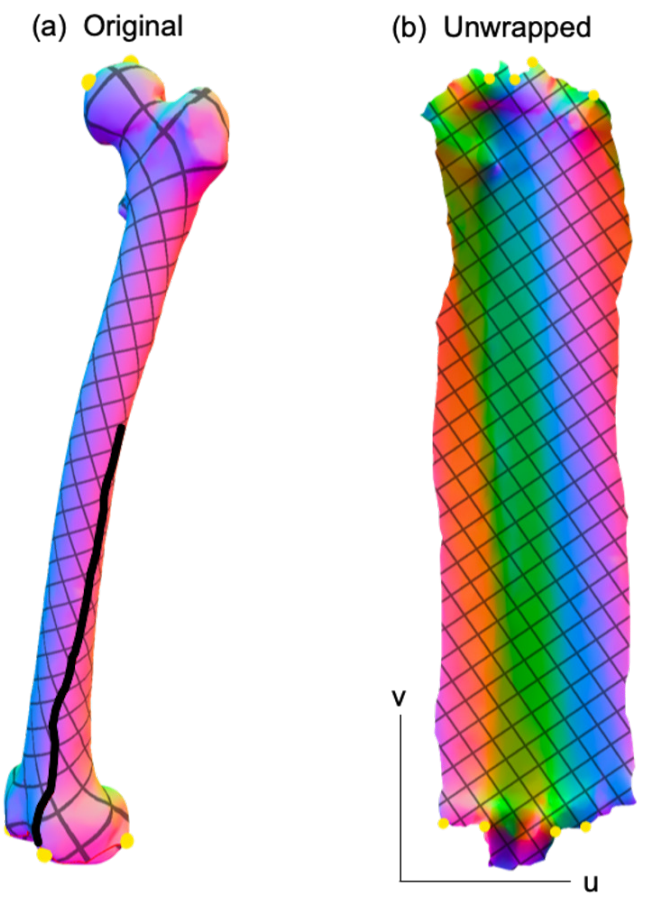

Figure 10. Example 3D surface unwrapping. (a) Original 3D geometry. (b) Unwrapped geometry; this is a $2 \mathrm{D}$ parametric (UV) representation of the original geometry. Colors represent changes in surface normal direction. The thick black line in panel (a) represents a seam along which the 3D geometry is cut so that it can be flattened into a 2D shape. Unwrapping was performed here using boundary first flattening (Sawhney and Crane, 2017).

just to point ordering. The current paragraph considers just one example (point ordering) as a potential source of sensitivity concern. In (Fig.11), the qualitative change in results can be attributed to a minor shift in point correspondence (Fig.11a-b), which created a small shift in pointwise covariance, but a shift that was large enough to alter the hypothesis rejection decision at $\alpha=0.05$. That is, point-specific covariance is direction dependent, so small changes in point-deformation direction can yield qualitative changes in test statistics (Pataky et al., 2014). Nevertheless, we observed this type of sensitivity to random point ordering only occasionally, with most randomizations resulting in qualitatively similar results. Also, in most cases we noticed that probability results, while variable, were generally stable. The problem only emerged qualitatively when that variability spanned $\alpha=0.05$, as depicted in Fig.11). This problem of probability value variability (Halsey et al., 2015) partially reflects a weakness of classical hypothesis testing, which has a binary interpretation of continuous probability. We acknowledge that we did not systematically conduct sensitivity testing, and also that each stage of processing involves a variety of components or parameters that could be subjected to sensitivity analysis. Comprehensive consideration of this sensitivity would require a large research effort, so we leave this for future work.

The datasets and analyses presented in this paper also have limitations. We analyzed shapes from just one database (Carlier et al., 2016) and, for each dataset, we selected only ten shapes for analysis, and only conducted two-sample tests. While we do not expect analysis of datasets from other databases to appreciably affect this paper's messages, we acknowledge that analyses of relatively small samples, and just one simple experimental design, fully exposes neither the advantages nor disadvantages of the proposed analysis framework. We selected just ten shapes for each dataset primarily to emphasize that the proposed parametric procedure is sufficiently sensitive to detect morphological effects for small sample sizes. The specific ten shapes were selected in an ad hoc manner to emphasize particular concepts including, for example: interpretation agreement between the proposed and landmark methods' results, and the opposite: interpretation disagreement. Since these datasets were selected in an ad hoc manner, from a single database, and with only two-sample analyses, the reader is left to judge the relevance of these results to other datasets and experimental designs. 

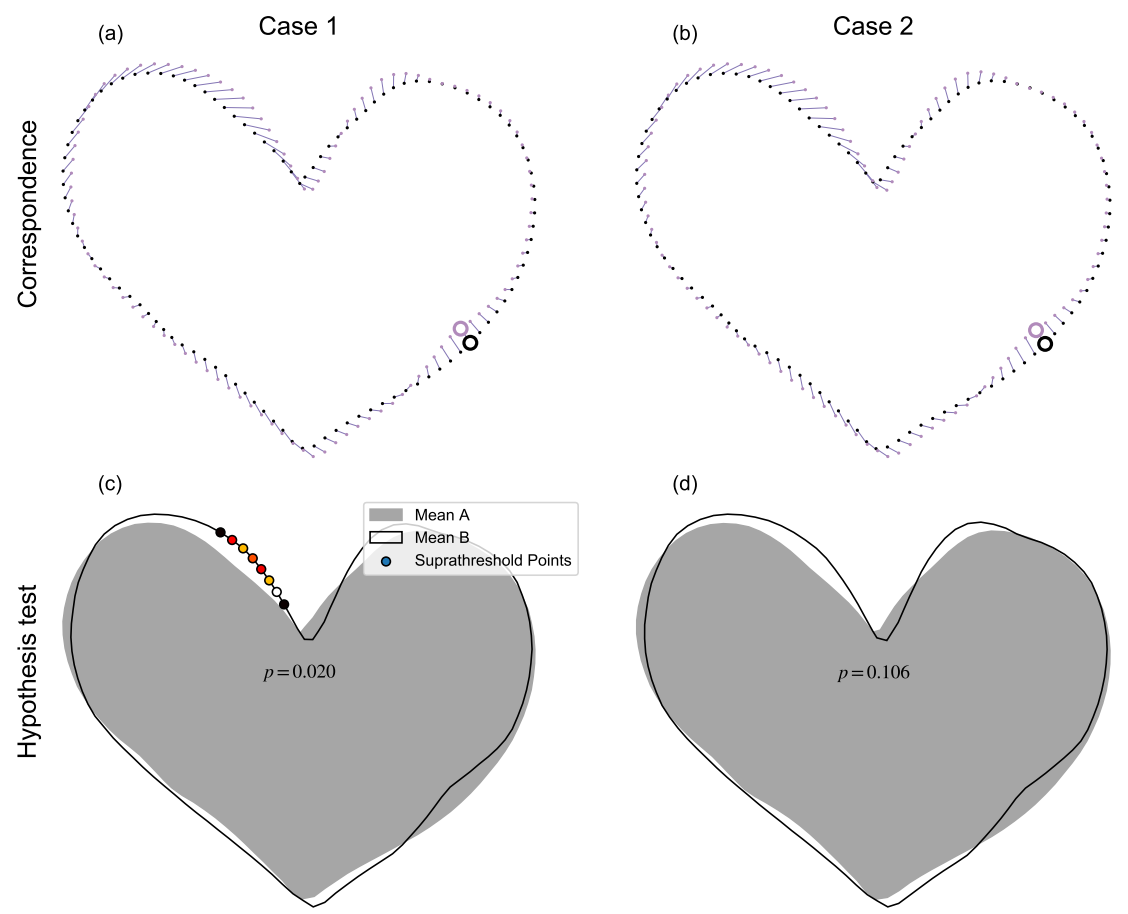

Figure 11. Example processing sensitivity. Case 1 depicts the result reported in Fig.8g. Case 2 depicts the results after point re-shuffling (i.e., a new random points order, see Fig.5a), then re-application of the processing chain depicted in Fig.1d. Note: results for Case 1 were qualitatively replicated for most random re-shufflings, but approximately 1 in 20 re-shufflings yielded qualitatively different results, like those depicted for Case 2 .

\section{CONCLUSIONS}

This paper demonstrates that parametric hypothesis testing can be conducted at the whole-contour level with suitably high statistical power for the analysis of even relatively small samples of 2D shapes $(N=10)$. We describe a general framework for automated, landmark-free hypothesis testing of $2 \mathrm{D}$ contour shapes, but this paper implements just one realization of that framework. The main advantages of the proposed framework are that results are calculated quickly ( $<2 \mathrm{~s}$ in this paper), and yield morphologically rich results in an easy-to-interpret manner. Since innumerable realizations of the proposed framework are possible through algorithm and parameter substitution at each stage in the proposed data processing chain, sensitivity analysis may generally be required for robust statistical conclusions.

\section{ACKNOWLEDGMENTS}

This work was supported by Kiban B Grant 17H02151 from the Japan Society for the Promotion of Science. There was no additional external funding received for this study.

\section{REFERENCES}

Adams, D. C. and Otárola-Castillo, E. (2013). Geomorph: an R package for the collection and analysis of geometric morphometric shape data. Methods in Ecology and Evolution, 4(4):393-399.

Adams, D. C., Rohlf, F. J., and Slice, D. E. (2004). Geometric morphometrics: ten years of progress following the 'revolution'. Italian Journal of Zoology, 71(1):5-16.

Adams, D. C., Rohlf, F. J., and Slice, D. E. (2013). A field comes of age: geometric morphometrics in the 21st century. Hystrix, 24(1):7.

Adler, R. J. and Taylor, J. E. (2007). Random Fields and Geometry. Springer-Verlag.

Anaconda (2020). Anaconda Software Distribution version 3-6.10. 
Anderson, M. and Braak, C. T. (2003). Permutation tests for multi-factorial analysis of variance. Journal of Statistical Computation and Simulation, 73(2):85-113.

Arnqvist, G. and Martensson, T. (1998). Measurement error in geometric morphometrics: empirical strategies to assess and reduce its impact on measures of shape. Acta Zoologica Academiae Scientiarum Hungaricae, 44(1-2):73-96.

Bingol, O. R. and Krishnamurthy, A. (2019). NURBS-Python: An open-source object-oriented NURBS modeling framework in Python. SoftwareX, 9:85-94.

Bonhomme, V., Picq, S., Gaucherel, C., and Claude, J. (2014). Momocs: Outline Analysis Using R. Journal of Statistical Software, 56(13):1-25.

Bookstein, F. L. (1996). Biometrics, biomathematics and the morphometric synthesis. Bulletin of Mathematical Biology, 58(2):313.

Bookstein, F. L. (1997). Landmark methods for forms without landmarks: morphometrics of group differences in outline shape. Medical Image Analysis, 1(3):225-243.

Brombin, C. and Salmaso, L. (2009). Multi-aspect permutation tests in shape analysis with small sample size. Computational Statistics \& Data Analysis, 53(12):3921-3931.

Carlier, A., Leonard, K., Hahmann, S., Morin, G., and Collins, M. (2016). The 2D shape structure dataset: a user annotated open access database. Computers \& Graphics, 58:23-30.

Casanovas-Vilar, I. and Van Dam, J. (2013). Conservatism and adaptability during squirrel radiation: what is mandible shape telling us? PLoS One, 8(4):e61298.

Chung, M. K., Worsley, K. J., Nacewicz, B. M., Dalton, K. M., and Davidson, R. J. (2010). General multivariate linear modeling of surface shapes using SurfStat. NeuroImage, 53(2):491-505.

Claes, P., Walters, M., Vandermeulen, D., and Clement, J. G. (2011). Spatially-dense 3d facial asymmetry assessment in both typical and disordered growth. Journal of anatomy, 219(4):444-455.

Claude, J. (2013). Log-Shape Ratios, Procrustes Superimposition, Elliptic Fourier Analysis: Three Worked Examples in R. Hystrix, the Italian Journal of Mammalogy, 24(1):94-102.

Collyer, M. L., Sekora, D. J., and Adams, D. C. (2015). A method for analysis of phenotypic change for phenotypes described by high-dimensional data. Heredity, pages 1-9.

Corti, M. (1993). Geometric morphometrics: an extension of the revolution. Trends in Ecology \& Evolution, 8(8):302.

Cullen, J. A. and Marshall, C. D. (2019). Do sharks exhibit heterodonty by tooth position and over ontogeny? a comparison using elliptic fourier analysis. Journal of Morphology, 280(5):687-700.

Da Costa, L. d. F. and Cesar, R. M. (2000). Shape Analysis and Classification: Theory and Practice. CRC Press, Inc.

Dale, A. M., Fischl, B., and Sereno, M. I. (1999). Cortical surface-based analysis: I. segmentation and surface reconstruction. Neuroimage, 9(2):179-194.

Devine, J., Aponte, J. D., Katz, D. C., Liu, W., Lo Vercio, L. D., Forkert, N. D., Marcucio, R., Percival, C. J., and Hallgrimsson, B. (2020). A Registration and Deep Learning Approach to Automated Landmark Detection for Geometric Morphometrics. Evolutionary Biology, 47(3):246-259.

Dumont, M., Wall, C. E., Botton-Divet, L., Goswami, A., Peigné, S., and Fabre, A.-C. (2016). Do functional demands associated with locomotor habitat, diet, and activity pattern drive skull shape evolution in musteloid carnivorans? Biological Journal of the Linnean Society, 117(4):858-878.

Friston, K. J., Ashburner, J. T., Kiebel, S. J., Nichols, T. E., and Penny, W. D. (2007). Statistical Parametric Mapping: The Analysis of Functional Brain Images. Elsevier, London.

Fruciano, C. (2016). Measurement error in geometric morphometrics. Development Genes and Evolution, 226(3):1-20.

Fu, A. L., Hammerschlag, N., Lauder, G. V., Wilga, C. D., Kuo, C.-Y., and Irschick, D. J. (2016). Ontogeny of head and caudal fin shape of an apex marine predator: The tiger shark (g aleocerdo cuvier). Journal of Morphology, 277(5):556-564.

Goodall, C. (1991). Procrustes methods in the statistical analysis of shape. Journal of the Royal Statistical Society: Series B (Methodological), 53(2):285-321.

Gower, J. C. (1975). Generalized procrustes analysis. Psychometrika, 40(1):33-51.

Hallgrimsson, B., Percival, C. J., Green, R., Young, N. M., Mio, W., and Marcucio, R. (2015). Morphometrics, 3D Imaging, and Craniofacial Development. In Craniofacial Development, pages 561-597. Elsevier.

Halsey, L. G., Curran-Everett, D., Vowler, S. L., and Drummond, G. B. (2015). The fickle P value 
generates irreproducible results. Nature Methods, 12(3):179-185.

Hill, J. J., Puttick, M. N., Stubbs, T. L., Rayfield, E. J., and Donoghue, P. C. (2018). Evolution of jaw disparity in fishes. Palaeontology, 61(6):847-854.

Holden, M. (2008). A review of geometric transformations for nonrigid body registration. IEEE Transactions on Medical Imaging, 27(1):111-128.

Kendall, D. G. (1977). The diffusion of shape. Advances in applied probability, 9(3):428-430.

Kendall, D. G. (1984). Shape manifolds, procrustean metrics, and complex projective spaces. Bulletin of the London mathematical society, 16(2):81-121.

Kendall, D. G. (1985). Exact distributions for shapes of random triangles in convex sets. Advances in Applied Probability, pages 308-329.

Kent, J. T. (1994). The complex bingham distribution and shape analysis. Journal of the Royal Statistical Society: Series B (Methodological), 56(2):285-299.

Klingenberg, C. P. and McIntyre, G. S. (1998). Geometric morphometrics of developmental instability: analyzing patterns of fluctuating asymmetry with procrustes methods. Evolution, 52(5):1363-1375.

Kurnianggoro, L., Wahyono, and Jo, K.-H. (2018). A survey of 2D shape representation: Methods, evaluations, and future research directions. Neurocomputing, 300:1-16.

Loy, A., Busilacchi, S., Costa, C., Ferlin, L., and Cataudella, S. (2000). Comparing geometric morphometrics and outline fitting methods to monitor fish shape variability of diplodus puntazzo (teleostea: Sparidae). Aquacultural Engineering, 21(4):271-283.

Mitteroecker, P. and Gunz, P. (2009). Advances in geometric morphometrics. Evolutionary Biology, 36(2):235-247.

Mitteroecker, P., Gunz, P., Bernhard, M., Schaefer, K., and Bookstein, F. L. (2004). Comparison of cranial ontogenetic trajectories among great apes and humans. Journal of Human Evolution, 46(6):679-698.

Morgan, C. C. (2009). Geometric morphometrics of the scapula of south american caviomorph rodents (rodentia: Hystricognathi): form, function and phylogeny. Mammalian Biology, 74(6):497-506.

Morris, B. (2003). The components of the wired spanning forest are recurrent. Probability theory and related fields, 125(2):259-265.

Murphy-Chutorian, E. and Trivedi, M. M. (2008). Head pose estimation in computer vision: A survey. IEEE transactions on Pattern Analysis and Machine Intelligence, 31(4):607-626.

Myronenko, A. and Song, X. (2010). Point set registration: coherent point drift. IEEE Transactions on Pattern Analysis and Machine Intelligence, 32(12):2262-2275.

Nichols, T. and Holmes, A. (2002). Nonparametric permutation tests for functional neuroimaging: a primer with examples. Human Brain Mapping, 15(1):1-25.

Nyquist, H. (1928). Certain topics in telegraph transmission theory. Transactions of the American Institute of Electrical Engineers, 47(2):617-644.

Page, C. E. and Cooper, N. (2017). Morphological convergence in 'river dolphin'skulls. PeerJ, 5:e4090.

Pataky, T. C. (2012). One-dimensional statistical parametric mapping in Python. Computer Methods in Biomechanics and Biomedical Engineering, 15(3):295-301.

Pataky, T. C., Caravaggi, P., Savage, R., and Crompton, R. (2008). Regional peak plantar pressures are highly sensitive to region boundary definitions. Journal of Biomechanics, 41(12):2772-2775.

Pataky, T. C., Robinson, M. A., Vanrenterghem, J., Savage, R., Bates, K. T., and Crompton, R. H. (2014). Vector field statistics for objective center-of-pressure trajectory analysis during gait, with evidence of scalar sensitivity to small coordinate system rotations. Gait and Posture, 40(1):255-258.

Pedoia, V., Samaan, M. A., Inamdar, G., Gallo, M. C., Souza, R. B., and Majumdar, S. (2017). Study of the interactions between proximal femur $3 \mathrm{~d}$ bone shape, cartilage health, and biomechanics in patients with hip Osteoarthritis. Journal of Orthopaedic Research, 25(1):114-12.

R Core Team (2019). R: A Language and Environment for Statistical Computing. R Foundation for Statistical Computing, Vienna, Austria.

Ramsay, J. O. and Li, X. (1998). Curve registration. Journal of the Royal Statistical Society Series B.

Renaud, S., Michaux, J., Schmidt, D. N., Aguilar, J. P., Mein, P., and Auffray, J. C. (2005). Morphological evolution, ecological diversification and climate change in rodents. Proceedings of the Royal Society B: Biological Sciences, 272(1563):609-617.

Rohlf, F. J. (1990). Fitting curves to outlines. In Proceedings of the Michigan morphometrics workshop, number 2, pages 167-177. The University of Michigan Museum of Zoology Ann Arbor Michigan.

Rohlf, F. J. (1999). Shape statistics: Procrustes superimpositions and tangent spaces. Journal of 
Classification, 16(2):197-223.

Rohlf, F. J. (2000a). On the use of shape spaces to compare morphometric methods. Hystrix-the Italian Journal of Mammalogy, 11(1).

Rohlf, F. J. (2000b). Statistical power comparisons among alternative morphometric methods. American Journal of Physical Anthropology: The Official Publication of the American Association of Physical Anthropologists, 111(4):463-478.

Rohlf, F. J. (2003). Bias and error in estimates of mean shape in geometric morphometrics. Journal of Human Evolution, 44(6):665-683.

Rohlf, F. J. and Marcus, L. F. (1993). A revolution morphometrics. Trends in ecology \& evolution, 8(4):129-132.

Sawhney, R. and Crane, K. (2017). Boundary first flattening. ACM Transactions on Graphics (ToG), 37(1):1-14.

Singleton, M. (2015). Functional geometric morphometric analysis of masticatory system ontogeny in papionin primates. The Anatomical Record, 298(1):48-63.

Slice, D. E. (2007). Geometric morphometrics. Annual Review of Anthropology, 36:261-281.

Stayton, C. T. (2005). Morphological evolution of the lizard skull: a geometric morphometrics survey. Journal of Morphology, 263(1):47-59.

Strait, J. and Kurtek, S. (2016). Bayesian model-based automatic landmark detection for planar curves. In Proceedings of the IEEE Conference on Computer Vision and Pattern Recognition Workshops, pages 86-94.

Taylor, J. E. and Worsley, K. J. (2008). Random fields of multivariate test statistics, with applications to shape analysis. Annals of Statistics, 36(1):1-27.

Terhune, C. E., Cooke, S. B., and Otárola-Castillo, E. (2015). Form and function in the platyrrhine skull: A three-dimensional analysis of dental and tmj morphology. The Anatomical Record, 298(1):29-47.

Toro-Ibacache, V., Muñoz, V. Z., and O'Higgins, P. (2016). The relationship between skull morphology, masticatory muscle force and cranial skeletal deformation during biting. Annals of AnatomyAnatomischer Anzeiger, 203:59-68.

van Rossum, G. (2019). The Python Library Reference Release 3.6.10.

Wuhrer, S., Shu, C., and Xi, P. (2011). Landmark-free posture invariant human shape correspondence. The Visual Computer, 27(9):843-852.

Young, R. and Maga, A. M. (2015). Performance of single and multi-atlas based automated landmarking methods compared to expert annotations in volumetric microCT datasets of mouse mandibles. Frontiers in Zoology, pages 1-12.

Zelditch, M. L., Swiderski, D. L., and Sheets, H. D. (2012). Geometric Morphometrics for Biologists: A Primer. Academic Press.

Zhang, D. and Lu, G. (2004). Review of shape representation and description techniques. Pattern Recognition, 37(1):1-19. 